\title{
Féeries
}

Études sur le conte merveilleux, XVII $-\mathrm{XIXe}$ siècle

$8 \mid 2011$

Le merveilleux français à travers les siècles, les langues, les continents

\section{Du salon littéraire à la chambre d'enfant. Réécritures des contes de fées français aux Pays-} Bas

From the Literary Salon to the Child's Bedroom. Rewritings of Fairy Tales in The Netherlands

\section{Daphne M. Hoogenboezem}

\section{OpenEdition}

\section{Journals}

Édition électronique

URL : http://journals.openedition.org/feeries/799

DOI : $10.4000 /$ feeries.799

ISSN : 1957-7753

\section{Éditeur}

UGA Éditions/Université Grenoble Alpes

\section{Édition imprimée}

Date de publication : 15 octobre 2011

Pagination : 91-116

ISBN : 978-2-84310-211-0

ISSN : $1766-2842$

\section{Référence électronique}

Daphne M. Hoogenboezem, « Du salon littéraire à la chambre d'enfant. Réécritures des contes de fées français aux Pays-Bas », Féeries [En ligne], 8 | 2011, mis en ligne le 15 avril 2013, consulté le 07 septembre 2020. URL : http://journals.openedition.org/feeries/799; DOI : https://doi.org/10.4000/ feeries.799 
Daphne M. Hoogenboezem

Université de Groningue (Pays-Bas)

\section{DU SALON LITTÉRAIRE À LA CHAMBRE D'ENFANT. RÉÉCRITURES DES CONTES DE FÉES FRANÇAIS AUX PAYS-BAS}

A VOGUE DU CONTE DE FÉES LITTÉRAIRE, lancée dans les salons parisiens dans la dernière décennie du XviI ${ }^{e}$ siècle, ne se limite pas à la France, bien au contraire. Aussitôt après la parution des éditions princeps des contes de Charles Perrault et de Marie-Catherine d'Aulnoy, leurs recueils sont publiés, souvent en contrefaçon, à l'étranger et notamment aux Pays-Bas. Ainsi, l'éditeur Adrian Moetjens publie deux contes en vers de Perrault dans le Recueil de pièces curieuses et nouvelles (La Haye, 1694). Les Histoires ou contes $d u$ temps passé paraissent dans le même recueil dès I697. Deux contrefaçons des contes en prose de Perrault paraissent en I697 et en I698 (Amsterdam, [Jacques Desbordes]) et Les Contes des fées de Madame d'Aulnoy sont publiés par Meindert Uytwerf en 1698 et en I700 (La Haye).

Ces premières éditions des contes sont entièrement en français et elles sont destinées à un public adulte de Français (vivant aux Pays-Bas ou bien en France) et de lecteurs cultivés néerlandais. Or, à partir des années I730, les contes de fées français paraissent également en néerlandais. Les contes de Madame d'Aulnoy sont traduits les premiers (Rotterdam, Jan Daniel Beman, I730). La première traduction des contes de Perrault paraît en I754 (La Haye, Pierre van Os). Ce changement de langue semble impliquer un changement de public. Les deux éditions sont adressées de manière explicite à un public jeune dans un but pédagogique. Les contes de Perrault sont intégrés immédiatement dans le domaine naissant de la littérature enfantine et, dans la seconde moitié du XVIII ${ }^{\mathrm{e}}$ siècle, les éditions du recueil, d'abord bilingues (français et néerlandais) puis exclusivement en néerlandais, se multiplient. Du recueil de Madame d'Aulnoy, en revanche, plus aucune traduction néerlandaise ne parait au XVIII ${ }^{\mathrm{e}}$ siècle. Les contes de Madame d'Aulnoy n'entrent pas dans le répertoire enfantin et ce n'est qu'au XIX ${ }^{\mathrm{e}}$ siècle qu'ils sont traduits à nouveau. Agatha (pseudonyme de 
Reinoudina de Goeje, I833-I893), une féministe déclarée et auteur de nombreux livres pour enfants, s'empare du recueil de la salonnière et réécrit une sélection de sept contes qui paraissent dans son recueil Sprookjes uit de oude doos (Contes d'hier, Tiel, Campagne, I882) ${ }^{\mathrm{I}}$.

Nous proposons d'analyser les (para)textes des deux traductions néerlandaises de Madame d'Aulnoy (I730 et I882) pour voir comment ses contes de fées, souvent considérés comme proto-féministes par la critique moderne américaine ${ }^{2}$, ont été traduits et adaptés par cette féministe pour le public néerlandais. Nous comparerons les traductions des contes de Madame d'Aulnoy à celles des contes de Perrault (I754, I780 et I790). Ceci nous permettra de mieux comprendre la réception des contes de fées français aux Pays-Bas.

\section{Le conte de fées et l'enfance}

Dans les contes de fées français, les références à l'enfance sont multiples. Dans la préface des contes en vers, Perrault associe ses contes à "ceux que nos aïeux ont inventés pour leurs enfants ${ }^{3} »$. Ces contes se distinguent des fables antiques par le fait qu'ils comportent tous une moralité «louable et instructive $^{4} »$. Il met en pratique cette vision dans ses Histoires ou contes $d u$ temps passé. Les huit contes en prose sont écrits dans un style simple qui s'approche du langage oral et ils sont suivis de moralités. Dans ses contes plus longs, Madame d'Aulnoy évoque le monde de l'enfance entre autres en mettant en scène des jouets's. Des poupées et des marionnettes figurent dans les contes Le Prince Lutin, La Princesse Carpillon, La Princesse Printanière, La Princesse Rosette, Serpentin vert et Finette Cendron. Dans La Chatte blanche, le prince se déplace à toute allure sur un petit cheval

I. Peu d'études ont paru sur R. de Goeje, ou Agatha, comme on l'appelait le plus souvent. Le fait que j’ai pu consulter sa correspondance personnelle m’a permis de développer une meilleure compréhension des idées de l'auteur, et de la façon dont elles sont reflétées dans ses ouvrages. Je tiens à remercier Madame J. M. Logger, arrière-petite-fille du frère d'Agatha, M. J. de Goeje, de m’avoir donné accès aux documents conservés dans les archives familiales.

2. Quelques exemples de cette lecture féministe des contes de fées français se trouvent dans L. C. Seifert, Fairy Tales, Sexuality and Gender in France (I670-17I5), Cambridge, Cambridge University Press, 1996 et P. Hannon, Fabulous Identities: Women's Fairy Tales in Seventeenth-Century France, Amsterdam, Rodopi, 1998.

3. Ch. Perrault, Contes, J.-P. Collinet (éd.), Paris, Gallimard, I98I, p. 5I.

4. Id.

5. M. Manson, «Madame d'Aulnoy, les contes et le jouet", dans J. Perrot (éd.), Tricentenaire Charles Perrault. Les grands contes du XVII siècle et leur fortune littéraire, Paris, In Press, 1998, p. I43-I56.

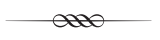


de bois. En outre la conteuse emploie plusieurs registres stylistiques, dont le langage enfantin. Dans La Princesse Rosette par exemple, l'hérö̈ne se réveillant trempée d'eau est prise de panique à l'idée d'avoir fait "pipi au dodo et d'être grondée ${ }^{6} »$. Or, malgré leur apparence naïve, ces contes n'ont pas été écrits pour des enfants, mais pour le public aristocratique et lettré des salons parisiens. Les références à l'enfance constituent une convention du genre. Elles font partie du jeu littéraire des auteurs qui cherchent à "créer des effets littéraires par la confrontation de registres narratifs et d'univers culturels aussi différents que possible», offrant aux lecteurs de l'époque une expérience de lecture inédite 7 .

Les premières éditions néerlandaises des contes de Perrault et de Madame d'Aulnoy sont, elles aussi, destinées à des adultes. Les éditeurs reprennent le texte français, et souvent aussi les lettres de dédicace et les préfaces, évoquant le monde raffiné des salons parisiens et de la cour. Quelquefois ils ajoutent également de nouvelles illustrations qui soulignent le caractère aristocratique et littéraire des contes. Ainsi, dans les premières éditions néerlandaises des Contes des fées de Madame d'Aulnoy (I698 et 1700), M. Uytwerf reprend le texte français, y compris la lettre de dédicace À Son Altesse Royale Madame, c'est-à-dire Élisabeth-Charlotte de Bavière ou la Princesse Palatine, l'épouse du frère du roi, et le «récit de Saint Cloud». Il fait graver des copies des vignettes originales d'Antoine Clouzier, mais il ajoute deux nouveaux frontispices. Ces gravures représentent des scènes de contage tout comme le célèbre frontispice de Perrault, mais dans la première illustration la conteuse populaire a été remplacée par une dame aristocratique, une salonnière peut-être, portant une belle robe et une fontange. Dans la seconde image, la conteuse paraît comme la déesse Minerve entourée de son public, l'illustre compagnie habillée au goût du jour décrite dans le «récit de Saint Cloud» qui ouvre le recueil ${ }^{8}$. Ces images sont réutilisées dans de nombreuses éditions ultérieures, dont

6. Voir J. Mainil, «Du triple usage de la parole dans le conte de la première vague : des huttes et des cabanes aux salons mondains", dans A. Defrance et J.-F. Perrin (éd.), Le Conte en ses paroles. La figuration de l'oralité dans le conte merveilleux du Classicisme aux Lumières, Paris, Desjonquères, 2007, p. 69.

7. J.-P. Sermain, Le Conte de fées du Classicisme aux Lumières, Paris, Desjonquères, 2005, p. 24 et 3I.

8. Nous pouvons être certains que ces deux frontispices ont été conçus exprès pour le recueil de Madame d'Aulnoy. Le frontispice représentant la conteuse comme une salonnière n'a pas été conçu pour l'édition des Illustres Fées de I698, comme le suggère G. Verdier dans «Les figures de la conteuse dans les contes de fées féminins" (XVII siècle, $\mathrm{n}^{\circ}$ I80, 1993, p. 489). La première contrefaçon néerlandaise des Contes des fées de Madame d'Aulnoy, publiée par M. Uytwerf en I698, comporte une épreuve inachevée de ce frontispice, qui a été retravaillée pour les éditions ultérieures, 
celles d'Estienne Roger (I708, I7II, I7I7, I719, I725). Les illustrations suggèrent que les éditions néerlandaises des contes français, et notamment celles de Madame d'Aulnoy, visent un lectorat adulte et lettré.

\section{Madame d'Aulnoy pour enfants?}

Cependant, en I730, paraît à Rotterdam, chez Jan Daniel Beman, un recueil de contes de fées intitulé La Vie des fées. Histoire pleine d'événements singuliers, étranges et très miraculeux, remplie de moralités utiles et de proverbes instructifs. Dépeinte de façon spirituelle et agréable. Mise à la disposition des amateurs des historiettes morales et en particulier de la jeunesse curieuse, pour les encourager de façon amusante à lire de bons livres; et surtout pour leur faire comprendre ce qui attend les personnes qui aspirent à la vertu, ainsi que le sort de ceux qui mènent leur vie autrement ${ }^{9}$. À en croire son long sous-titre, le recueil de J. D. Beman viserait principalement la jeunesse curieuse (weetgierige jeugd), sans pour autant exclure des lecteurs plus âgés qui seraient intéressés par des zedige snakerijen, c'est-à-dire des histoires drôles et morales. À ma connaissance, l'ouvrage est le premier recueil de contes de fées publié aux Pays-Bas à être adressé de façon explicite à un public jeune. Le fait que le livre soit destiné à la jeunesse est remarquable, puisque le public néerlandais aurait associé les contes de fées français plutôt aux adultes qu'aux enfants. L'édition de J. D. Beman est-elle le premier recueil de contes pour enfants (comme le suggère son incorporation dans l'étude sur les livres de jeunesse et manuels scolaires aux Pays-Bas de P. J. Buijnsters ${ }^{\mathrm{IO}}$ ), ou la référence à l'enfance est-elle une simple convention littéraire, comme dans les contes de fées français? Plusieurs indices, dont l'apparence luxueuse de l'édition et la traduction, laissent à penser que le recueil était en réalité destiné à un public adulte.

\footnotetext{
dont celle des Illustres Fées également publiée par M. Uytwerf en I698. La seconde contrefaçon néerlandaise de I700 contient une épreuve inachevée du frontispice avec la conteuse-Minerve.

9. [M.-C. d'Aulnoy], Het leven der tovergodinnen. Zynde een verhaal van zonderlinge, vreemde en zeer wonderlyke gevallen, vol van zinryke zedenlessen en leerzame spreuken. Alles zeer geestig en aardig beschreven. Ten dienste der liefhebbers van zedige snakeryen, en inzonderheid van de weetgierige jeugd, om ze op een vermakelyke wyze aan te sporen tot het lezen van goede boeken; ook voornamelyk om hen te doen beseffen wat ze te wachten hebben, die de deugd najagen, en wat't lot is van zodanige, die hun leven anders aanleggen, (Rotterdam, J. D. Beman, I730. Toutes les traductions sont les miennes.) Iо. P. J. Buijnsters et L. Buijnsters-Smets, Bibliografie van Nederlandse school - en kinderboeken I700 - I800, Zwolle, Waanders, I997, $\mathrm{n}^{\text {os }}$ I52I et I522.
} 
Le recueil est imprimé en format octavo, sur un papier de qualité et il est illustré de sept gravures sur cuivre en pleine page. Les images ne sont pas signées, mais elles semblent avoir été gravées exprès. Les scènes représentées correspondent très exactement au texte des contes et, à ma connaissance, elles ne paraissent dans aucune autre édition. Le livre était sans aucun doute assez cher. L'était-il trop pour un livre d'enfants? En tout cas les exemplaires consultés dans le cadre de cette recherche ne portent point de traces de lecture par un public jeune ${ }^{\text {II }}$. Le prix et le tirage sont inconnus. Le recueil a été publié aux frais du traducteur, mais son nom ainsi que ceux des auteurs des contes qui y sont inclus manquent. L'édition comporte douze contes dont quatre sont de Madame d'Aulnoy, à savoir De spokende prins (Le Prince Lutin), De gele dwerg (Le Nain jaune), De blauwe vogel (L'Oiseau bleu) et De gouden tak (Le Rameau d'or). Ces quatre contes proviennent du premier recueil de Madame d'Aulnoy, Les Contes des fées (1697), qui avait été réédité plusieurs fois en France. Le traducteur a pu se baser également sur l'une des éditions néerlandaises de M. Uytwerf ou de E. Roger. En outre, le recueil comporte une sélection de sept contes provenant du recueil Les Illustres Fées, publié de manière anonyme en $1698^{12}$. Nous savons actuellement que son auteur est le Chevalier Louis de Mailly. Cependant, aux XVII ${ }^{\mathrm{e}}$ et XVIII ${ }^{\mathrm{e}}$ siècles, l'ouvrage est souvent attribué à Madame d'Aulnoy. Dès i698, l'éditeur M. Uytwerf en publie une édition à laquelle il ajoute le nom de Madame $\mathrm{D}^{* * *}$, abréviation que Madame d'Aulnoy emploie pour signer ses ouvrages, ainsi que le frontispice représentant la salonnière, qu'il avait utilisé pour l'édition de Madame d'Aulnoy. Il s'agit probablement d'une manœuvre publicitaire de la part de M. Uytwerf, mais l'attribution fautive est reprise par de nombreux éditeurs ultérieurs, dont E. Roger, qui ajoute le recueil des Illustres Fées à deux compilations publiées en I7II et en I7I7. Il est donc probable que J. D. Beman pensait que ces sept contes étaient de Madame d'Aulnoy.

Le recueil de J. D. Beman comporte enfin un conte de Marie-Jeanne Lhéritier, Behendige, of de schrandere prinses (Les Aventures de Finette ou l'Adroite Princesse). Ce conte paraît en 1695 dans les Euvres mêlées. Aucune édition complète de cette œuvre de Mademoiselle Lhéritier n'a été publiée aux Pays-Bas avant 1730, or dès I7I6 J. Desbordes intègre le

\footnotetext{
II. Trois exemplaires conservés aux bibliothèques universitaires d'Amsterdam et de Leyde. I2. Les sept contes provenant des Illustres Fées sont : De weldoener of Quiribirini (Le Bienfaisant ou Quiribirini), De koningin van het bloemeneiland (La Reine de l'Ile des Fleurs), Fortunio (Fortunio), Gunsteling der Tover-godinnen (Le Favori des fées), Koning Toveraar (Le Roi Magicien), Prins Rogier (Le Prince Roger), De Prinses gekroond door de Tover-Godinnen (La Princesse couronnée par les fées).
} 
conte L'Adroite Princesse dans ses éditions des Histoires ou contes du temps passé de Perrault. Le conte paraît dans la plupart des éditions ultérieures des contes de Perrault (I72I, I729), ainsi que dans le Cabinet des fées de E. Roger (I7I7), donnant lieu à une tradition de fausses attributions que l'on retrouve encore dans certaines éditions aujourd'hui.

Les douze contes que J. D. Beman sélectionne pour son recueil ne conviennent peut-être pas tous à un public jeune. L'auteur ne précise point la classe d'âge qu'il vise. La traduction néerlandaise pourrait être adressée à des enfants, mais au XVIII ${ }^{\mathrm{e}}$ siècle les adolescents sont également appelés jeugd («jeunesse») ${ }^{13}$. Or l'instruction morale annoncée dans le sous-titre est douteuse. Madame d'Aulnoy met en scène des princesses actives et intelligentes. La princesse Toute Belle, héroïne du conte Le Nain jaune, refuse de se marier et, dans L'Oiseau bleu, la princesse Florine part toute seule à la recherche de son amant. Ces personnages féminins trouvent leur place dans le débat sur le statut de la femme dans la société qui anime les salons parisiens de la fin du XVII ${ }^{\mathrm{e}}$ siècle mais, au XVIII ${ }^{\mathrm{e}}$ siècle, ces princesses exceptionnelles n'étaient peut-être pas considérées comme des modèles pour les jeunes filles. Et que penser du méchant prince dans Les Aventures de Finette qui couche avec les deux sœurs de l'héroïne sous prétexte de les épouser le lendemain, ou du prince Roger qui, ayant découvert sa maîtresse avec un autre amant, brutalise son rival, lui casse les dents et le jette par la fenêtre?

\section{Les contes, de «bons livres»?}

Outre les douze contes de fées (dont onze étaient communément attribués à Madame d'Aulnoy), l'édition de J. D. Beman comporte une longue préface «au lecteur». Le préfacier y expose le double but de l'édition : l'éducation morale des lecteurs et l'incitation de la jeunesse à la lecture. Il explique que la lecture est un bon passe-temps qui empêchera les jeunes de $s^{\prime}$ ennuyer ${ }^{14}$. En outre, il considère la lecture des contes instructive et amusante pour les enfants. Le point de vue de J. D. Beman est remarquable parce qu'en France, comme aux Pays-Bas, les contes de fées sont parfois

\footnotetext{
I3. Voir P. J. Buijnsters, De hele Bibelebontse berg, De geschiedenis van het kinderboek in Nederland en Vlaanderen van de Middeleeuwen tot heden, Amsterdam, Querido, 1989, p. I7I.

I4. "[...] indien de jonge luiden zich wat naarstiger tot lezen begaven, ze zouden zomtyds zoo verlegen niet zyn met hunnen tyd, waaruit niets goeds voortkomt». ("Si les jeunes gens se consacraient avec plus d'application à la lecture, ils ne seraient pas aussi embarrassés de leur temps, dont il ne résulte rien de bon.») [M.-C. d'Aulnoy] (Het leven der tovergodinnen, ouvr. cité, p. 3)
}

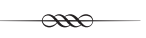


méprisés par des lecteurs cultivés, et déconseillés comme lecture pour la jeunesse. À la fin du XVII ${ }^{\mathrm{e}}$ siècle, l'abbé Pierre de Villiers critique le genre dans ses Entretiens sur les contes de fées et sur quelques autres ouvrages du temps, pour servir de préservatif contre le mauvais goût (I699). Aux Pays-Bas, l'attitude négative à l'égard des contes (populaires) dans l'éducation des enfants transparait dans les œuvres de Johan Van Heemskerk (I597-I656) et de Justus Van Effen (I684-I735) I5. Dans sa Batavische Arcadia (I637), J. Van Heemskerk incite les parents à raconter plutôt des histoires sur la révolte des Pays-Bas (I568-I648) que «des contes insipides» :

Oui on doit aux petits enfants de les [les histoires authentiques] leur donner, dès le berceau, comme à manger dans leur bouillie ou à boire dans leur bouteille. Et les mères et les nourrices devraient en raconter sans cesse à leurs élèves, au lieu des contes insipides de la Rood-Kousje, de la Smeer-bolletje ou des Singende springende Lovertjes etc. ${ }^{16}$

Il mentionne entre autres le conte Rood Kousje (Petite Chaussette rouge). Le contenu de cette histoire est aujourd'hui incertain, mais le titre paraît à plusieurs reprises dans d'autres textes de l'époque comme exemple type des contes que l'on raconte aux enfants aux Pays-Bas ${ }^{17}$. L'opinion ne semble pas avoir changé au début du XviII ${ }^{\mathrm{e}}$ siècle. Quelques années après la publication du recueil de J. D. Beman, J. Van Effen exprime des critiques comparables sur les contes dans le périodique Le Misanthrope (1745). Les deux auteurs préfèrent les récits historiques authentiques pour l'éducation des enfants, et condamnent les contes de fées mensongers. L'auteur de l'édition Beman semble se souvenir du texte de J. Van Heemskerk et se défend contre cette critique en écrivant :

Oui on peut dire qu'il est en quelque sorte mieux pour la jeunesse de lire des livres de cette nature [contes, histoires fictives], que de feuilleter des livres d'histoire, parce que l'histoire étant complètement sujette à la vérité, montre quelquefois des choses scandaleuses et immorales. L'histoire peint les hommes tels qu'ils sont, décrivant leurs faits et gestes, soit odieux, soit louables, tandis que ces livres mettent en scène les sujets tels qu'ils devraient être ${ }^{\mathrm{r}}$.

I5. P. J. Buijnsters, De hele Bibelebontse berg, ouvr. cité, p. 178.

16. "Iae men is 't schuldigh, de kleyne kinderen, vande wiegh aen, als in haer pap te eten, en in haar pyp-kan te drincken te gheven. En moeders, en minnens mosten 't haere Queeckelingen, in plaetse vande souteloose sproockjes, van 't Root-kousje, van 't Smeer-bolletje vande Singhende springende Lovertjes, en diergelycke, sonder ophouden vertellen, en inscherpen." (Voir J. Van Heemskerk, Batavische Arcadia, I637, dans G. J. Boekenoogen, "Nederlandse sprookjes uit de XVII" en het begin der XVIII ${ }^{\text {de }}$ eeuw", Volkskunde XX [1909], p. I30-I3I)

I7. G. J. Boekenoogen, ibid., p. I31-I32.

I8. "Ja, men kan zeggen, dat 't lezen van boeken van deze natuur [sprookjes, fictieve vertellingen] in zeker opzicht beter is voor de jeugd, dan 't doorbladeren van geschiedenissen, doordien geschiedenis 
Non seulement les contes peuvent être lus par la jeunesse, ils constitueraient même des lectures divertissantes, raisonnables et instructives.

\section{Contes médiévaux et proverbes}

De plus, J. D. Beman développe un discours sur la valeur éducative et littéraire des contes dans son recueil. Il soutient qu'ils sont instructifs puisqu'ils aident à expliquer les proverbes. En outre, ils font partie d'une tradition littéraire remontant au Moyen Âge. Cette théorie sur l'origine des contes de fées n'est pas originale, elle provient de Mademoiselle Lhéritier, la nièce de Perrault et principale théoricienne du genre avec lui. Elle écrit dans la lettre de dédicace à Madame D. G*** qui ouvre ses Euvres mêlées:

Pour moi ce que je me propose de vous dire, est que les troubadours sont les auteurs des petites histoires dont je vous ai parlé. [...] On voit de petites histoires répandues dans le monde, dont tout le dessein est de prouver agréablement la solidité des proverbes. Nos ancêtres, qui étaient ingénieux dans leur simplicité, s’apercevant que les maximes les plus sages s'impriment mal dans l'esprit, si on les présente toutes nues, les habillèrent, pour parler ainsi, et ils les firent paraître sous des ornements. Ils les exposèrent dans de petites histoires qu'ils inventèrent, ou dans le récit de quelques événements qu'ils embellirent; et comme ces récits n'avaient pour but que l'instruction des jeunes gens, et qu'il n'y a que le merveilleux qui frappe bien vivement l'imagination, ils n'en furent pas avares; les prodiges sont fréquents dans leurs fables ${ }^{19}$.

L'auteur de l'édition Beman la cite presque littéralement ${ }^{20}$, pourtant il ne renvoie pas à Mademoiselle Lhéritier mais reprend sa référence au savant homme (geleerde), c'est-à-dire Pierre Daniel Huet. En effet dans sa

ganschelyk aan de waarheid onderheven, somtyds aanstotelyke zedeloze dingen vertoont, de geschiedenis schildert de menschen, zoo alsze zyn, beschryvende dezelver daden en bedryven, 't zy verfoeilyk of pryswaardig, deze boeken daarentegen doen de voorwerpen ten tonele verschynen zoo alsze behoorden te zyn." [M.-C. d'Aulnoy], (Het leven der tovergodinnen, ouvr. cité, p. 3-4)

I9. M.-J. Lhéritier, Contes, Paris, Champion, 2005, p. 36. Nous soulignons.

20. "Om ook iets van den oorsprong den verdichte geschiedenissen te zeggen: 'T gansche oogmerk van dezelve is, om op eene aangename wyze de degelykheid der spreekwoorden aan te tonen. Onze voor ouders die geestryk waren in hunne eenvoudigheid, bespeurende dat zelfs de zinryke grondspreuken waarna wy onzen handel en wandel behoren te schoeien verkeerde denkbeelden geven als men ze gansch naakt in de wereld zend, hebben dezelve om zo te spreken gekleed en onder versieringen doen verschynen; ze lieten dezelve invloeien in vertellingjes, welke zy verzonnen, of in 't verhaal van enige geschiedenissen, die ze zo veel mogelyk daarmede opschikten. En doordien diergelyke vertellingen enkelyks strekten ter onderwyzingen van de jeugd, en niets zoo een levendige indruk op het gemoed maakt, dan onbegrypelijke, verwonderenswaardige gevallen, zo zyn ze daaromtrent niet karig geweest. [...] Zeker geleerde zegt in zyn vertoog over deen oorsprong van de verdichte geschiedenissen, dat de Troubadours de eerste schryvers van dezelve geweest zijn." [M.-C. d'Aulnoy] (Het leven der tovergodinnen, ouvr. cité, p. 5-6. Nous soulignons) 
lettre, Mademoiselle Lhéritier renvoie à la Lettre à Monsieur de Segrais sur l'origine des romans (I682) de P. D. Huet, qu'on peut considérer comme la première histoire moderne du roman.

J. D. Beman emploie la théorie de Mademoiselle Lhéritier afin d'étayer la valeur éducative, morale et littéraire des contes. Aux onze contes attribués à Madame d'Aulnoy, il ajoute un conte de Mademoiselle Lhéritier, pour que la théorie développée dans la préface soit reflétée dans le texte des contes. Le conte L'Adroite Princesse est situé au temps des premières croisades. En outre, Mademoiselle Lhéritier y met en pratique la théorie des proverbes. Elle dédicace ce conte à la conteuse Henriette-Julie de Murat. Dans la lettre qui précède le conte, elle explique qu'il illustre deux proverbes, à savoir "Oisiveté est mère de tous vices» et "Défiance est mère de sureté». J. D. Beman omet la lettre de dédicace, mais il mentionne l'équivalent de la première expression dans la préface : Een lui mens is een duivelsoorkussen ("Un homme paresseux est un oreiller pour le diable»).

Cependant la théorie de Mademoiselle Lhéritier semble être reflétée également dans la composition de la collection et dans la traduction. En effet, J. D. Beman sélectionne plusieurs contes situés dans un cadre vaguement médiévisant. La princesse Florine, héroïne du conte L'Oiseau bleu, est emprisonnée dans un donjon. Dans Le Nain jaune, Madame d'Aulnoy évoque le palais magique du nain, et dans Le Rameau d'or le prince Torticolis, emprisonné dans une vieille tour, découvre des livres écrits dans un langage si ancien qu'il n'y comprend rien, un manuscrit précieux dont «les feuilles étaient de vélin, peintes tout autour, et la couverture d'or émaillé de bleu " et des vitres peintes de couleurs vives ${ }^{21}$.

De plus, le traducteur se montre sensible au jeu littéraire des auteurs français. Au lieu d'adapter les contes, parfois ambigus, pour un public jeune, il semble avoir voulu créer une expérience de lecture comparable à l'original français. Il traduit les contes français mot à mot ou presque. Les adaptations sont aussi rares que superficielles. Quelquefois il change le nom d'un personnage et il remplace des expressions par des équivalents néerlandais. Ainsi, les méchantes sœurs de la princesse Finette battent leur sœur «comme plâtre» dans la version française, alors que dans le texte néerlandais elles la battent "comme une merluche" (beuken als stockvisch). Dans le conte Le Prince Lutin, le méchant prince Furibond fait "une capilotade» de son rival. Le traducteur remplace cette sauce de viande probablement peu connue des lecteurs néerlandais par un plat plus

2I. Madame d'Aulnoy, Contes des fées, N. Jasmin (éd.), Paris, Champion, 2008, p. 295-297. 
familier : hutspot («purée de légumes»). Dans le même conte, une fée «fait le sabbat» à son mari infidèle. Le traducteur introduit alors une expression similaire néerlandaise, De Droes rijdt op stelten ( Le diable roule sur des échasses").

L'auteur de l'édition Beman ajoute également de nouvelles expressions, qui remplacent le texte français plus neutre. Dans le conte Le Prince Lutin, le prince Léandre sauve la vie d'une couleuvre, qui est en fait la fée Gentille. Il décide de garder l'animal et de le nourrir. Un jour, le serpent prend sa première forme et le héros découvre à sa place une belle dame. «Le jeune prince, qui n'avait point eu jusque-là de commerce avec les fées demeura si surpris qu'il fut longtemps sans pouvoir parler $^{22}$." Or, dans la version néerlandaise, le personnage "reste figé comme une statue de sel, sans pouvoir prononcer un $\operatorname{mot}^{23} »$. Outre cette expression d'origine biblique, on y trouve plusieurs autres expressions plus populaires. En récompense de son aide, la fée donne au prince un chapeau magique qui lui permet de se rendre invisible, et trois roses. L'une garantit la santé, la deuxième la richesse et la troisième lui permet de tester la fidélité de sa maîtresse. Au lieu d'une traduction littérale, l'auteur de l'édition Beman écrit qu'en mettant cette rose sur la poitrine de sa maitresse «il saura si le cheval boite ${ }^{24}$ ». Sans doute il s'agit d'une allusion à l'expression néerlandaise, "Le cheval boiteux arrive en dernier" (Het hinkend paard komt achteraan), qui avertit l'homme qui se réjouit trop tôt d'une bonne nouvelle qu'une mauvaise nouvelle peut suivre contre toute attente ${ }^{25}$. Dans le conte Le Rameau d'or, le prince Torticolis, enfermé dans un vieux donjon, trouve dans un cabinet la main d'un homme baignant dans le sang qui tient encore une boîte de portraits. "À cette vue Torticolis frémit, ses cheveux se hérissèrent», alors que dans la version néerlandaise "cela lui faisait monter les cheveux à la montagne ${ }^{26}$. L'emploi des expressions rend le texte plus vivant et atteste d'une certaine sensibilité pour le langage oral qui rappelle les jeux linguistiques des auteurs français qui emploient parfois des mots ou des tournures

\footnotetext{
22. Ibid., p. 205.

23. "Hy stond lang als een zoutpilaar, zonder een woord te kunnen voortbrengen.» [M.-C. d'Aulnoy] (Het leven der tovergodinnen, ouvr. cité, p. I2)

24. "Dat hy de tweede zyne minnares op de borst leggende zou weten of 't paard ook hinkte." (Ibid., p. 15$)$

25. F. A. Stoett, Nederlandsche spreekwoorden, spreekwijzen, uitdrukkingen en gezegden, W. J. Thieme \& Cie., Zutphen 1923-1925, dans Digitale Bibliotheek voor de Nederlandse Letteren en ligne : www.dbnl.org/tekst/stoeooznedeoI_oI/stoeoo2nedeoI_OI_I8I3.php (2 avril 20II).

26. "Op dit gezicht sidderde hy; de haren rezen hem te berge." [M.-C. d'Aulnoy] (Het leven der tovergodinnen, ouvr. cité, p. 399)
}

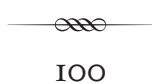


désuets, ou bien des expressions du langage populaire ou enfantin. Les proverbes et les expressions ajoutés dans le texte des contes font sans doute également écho à la théorie de Mademoiselle Lhéritier sur les proverbes.

Outre ces expressions, le traducteur ajoute quelques références nouvelles au monde de l'enfance. Dans le conte Le Prince Lutin, des ambassadeurs arrivant à la cour du roi, le père du prince Furibond, prennent le prince malformé pour son nain et ils le tournent et le retournent. Le traducteur écrit qu'ils le "faisaient tourner comme une toupie ${ }^{27}$ ", développant ainsi les références aux jouets employées par Madame d'Aulnoy. De plus, des enfants sont mentionnés plusieurs fois dans les contes. Ainsi le prince Torticolis, dans le conte Le Rameau d'or, est « un croque-mitaine qui aurait fait rentrer les enfants dans leurs coquilles» et, dans Le Prince Lutin, une fée rend ses rivales si terrifiantes qu'elles auraient "renvoyé les enfants au lit ${ }^{28}$. Ces quelques références à l'enfance suggèrent que le traducteur a voulu rebroder sur le jeu littéraire des conteurs français plutôt que d'adapter les contes au public enfantin.

Nous ignorons les réactions du public à cette édition. Cependant l'édition ne semble pas avoir été un succès de librairie. Nous ne connaissons que deux éditions du recueil, datant de 1730 et 1732 . Or lorsqu'on compare le texte des deux éditions, on retrouve les mêmes coquilles, et les marques de l'imprimeur en bas du texte - ce que l'on appelle l'empreinte d'un ouvrage - sont identiques. Il s'avère que l'éditeur de la seconde édition, Rudolf Brouwer, a seulement ajouté une nouvelle page de titre à l'édition princeps. Une réédition rapide est un signe de la réussite d'un ouvrage, mais l'insertion d'une nouvelle page de titre est souvent preuve d'un échec. Après deux années de ventes décevantes, J. D. Beman a sans doute vendu le reste du tirage à $\mathrm{R}$. Brouwer, à Amsterdam, qui a fait imprimer une nouvelle page de titre pour suggérer une édition nouvelle. Des rééditions ultérieures du recueil J. D. Beman manquent et aucune autre traduction des contes de Madame d'Aulnoy ne semble avoir été publiée au XVIII ${ }^{\mathrm{e}}$ siècle. Ceci montre que les contes de Madame d'Aulnoy n’ont pas été rangés dans la littérature enfantine, secteur de la librairie qui commence tout juste à se développer.

27. "Vervolgens den Prins Furibundus aankykende namen ze hem voor een dwerg, en draaiden hem als een tol, zoo dat hy van nyd meende uit zyn vel te springen." (Ibid., p. 4)

28. "T past $u$ byzonder, zeide de koning, gebreken in die princesse te vinden, zynde zelf een rechte bullebak, en goed om kinderen in hun schulpen te doen kruipen.» (Ibid., p. 399.) "Zoodra hy de minste genegenheid voor een ander bespeurde reed de droes by zynent op stelten, enze maakte de mooiste perzoon van de wereld zoo lelyk dan men kinderen daarmede na bed zou hebben gejaagd." (Ibid., p. 32)

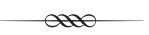




\section{Perrault en néerlandais}

La première traduction néerlandaise des contes de Perrault paraît dans l'édition bilingue intitulée Contes de ma Mère l'Oye, avec neuf gravures sur cuivre excellentes. Très utile à la jeunesse pour s'exercer au français et au hollandais $(\mathrm{I} 754)^{29}$. Le recueil est imprimé in octavo et illustré de neuf gravures sur cuivre pleine page, comme celui de J. D. Beman, mais il est moins épais (I50 pages seulement contre 462 dans l'édition Beman) et les illustrations (gravées par l'artiste néerlandais Simon Fokke) avaient déjà été utilisées dans d'autres éditions publiées en 1742 et en $1745^{30}$. Pour le recueil bilingue de $\mathbf{1 7 5 4}$, l'éditeur a peut-être repris le texte français d'une de ces éditions, cependant quelques-uns des archaïsmes et néologismes de Perrault ont été éliminés. Le mot «Mère-grand» est remplacé par la variante plus moderne "grand-mère» et la bûcheronne devient la femme du bûcheron. Cependant, le traducteur anonyme a su donner un charme naïf à la version néerlandaise des contes, qui est imprimée en caractères italiques en regard du texte français. Il emploie quelques archaïsmes (entre autres kaproentje pour "chaperon", krauwels pour "marmitons") et des expressions populaires ou enfantines. Le compère-loup devient wolf-neef ("loup-cousin») et l'ogresse dans La Belle au bois dormant est le plus souvent appelée ondeugende koningin ("reine espiègle») 31. La version néerlandaise est en général fidèle à l'original mais, par endroits, le traducteur adapte le texte au public néerlandais. Il remplace la formulette "Tire la chevillette, la bobinette cherra" par "Tire la cordelette, le loquet se lèvera", évoquant la mécanique des portes néerlandaises ${ }^{32}$. Quelquefois il ajoute des références aux croyances populaires hollandaises. Il adapte par exemple le début du conte Le Petit Poucet en écrivant :

Il était une fois un bûcheron et sa femme, qui avaient sept enfants, tous des garçons. L'aîné n'avait que dix ans et le cadet avait déjà sept ans. L'on s'étonnera que le

29. [Ch. Perrault], Contes de ma Mère l'Oye. Vertellingen van Moeder de Gans. Met negen keurlyke koopere plaatjes, zeer dienstig voor de Jeugdt om haar zelve in het Fransch en Hollands te oeffenen, La Haye, P. van Os, I754. L'édition comporte les huit contes en prose de Perrault.

30. Histoires ou contes du temps passé avec des moralités par M. Perrault. Nouvelle édition augmentée d'une nouvelle à la fin, La Haye, s. n., 1742 et Contes de ma Mère l'Oye - Mother Goosés Tales, La Haye, J. Neaulme, I745.

3I. Voir G. W. Wolthuis, «De Sprookjes van Moeder de Gans. V. De oudste Nederlandse vertalingen", Levende Talen, I947, p. 80.

32. Ibid., p. 8o. 
bûcheron ait eu autant d'enfants en si peu de temps, mais sa femme allait tous les ans en bateau au Volewijk et en ramenait en général deux à la fois ${ }^{33}$.

Le Volewijk (Quartier des oiseaux; Volen > Vogelen > Oiseaux) était un polder au nord d'Amsterdam où il y avait beaucoup d'oiseaux. À l'époque on y installait les gibets et les cadavres les attiraient beaucoup ${ }^{34}$. Curieusement, le Volewijk était désigné dans le folklore de l'époque comme l'endroit où pousseraient les petits enfants avant d'être "cueillis de l'arbre des enfants" par leurs parents. On trouve des références à ce conte de bonne femme entre autres dans l'histoire de Jan de Wasser («Jean le Laveur») et sa femme Griet. Dans cette histoire populaire portant sur la répartition des rôles, le mari accomplit toutes les tâches attribuées à la femme. C'est le monde à l'envers. L'histoire est très prisée à partir du $\mathrm{XVII}^{\mathrm{e}}$ jusqu'au XIX ${ }^{\mathrm{e}}$ siècle, et de nombreuses éditions de cette histoire en images sont publiées (pour adultes mais aussi pour la jeunesse). Parmi les illustrations, il en est une qui représente le couple se dirigeant au Volewijk en bateau, tandis que de l'autre côté on aperçoit déjà l'arbre des enfants ${ }^{35}$.

Enfin, le traducteur ajoute quelques explications pour son jeune public. La nuit de noces, la Belle au bois dormant n'a pas sommeil, "ayant dormi cent ans", et Cendrillon s'assoit dans les cendres "parce qu' elle est frileuse et qu'on ne lui permet pas de chauffe-pieds ${ }^{36}$. L'auteur rationnalise les contes. Il atténue le caractère légèrement érotique de la scène de la nuit de noces, et offre une explication nouvelle du comportement de Cendrillon qui accentue le mauvais traitement de la jeune héroïne. La première traduction a beaucoup de succès. Plusieurs rééditions d'abord bilingues puis exclusivement néerlandaises sont publiées, entre autres en 1759, I765, I775, 1784 et 1785 .

D'autres éditeurs, inspirés par le succès du recueil, publient de nouvelles traductions, souvent de moindre qualité, qui sont, elles aussi, destinées à un public jeune. Souvent elles paraissent dans des éditions modestes. Vers I780 paraît le recueil intitulé Contes amusants du temps passé, ou Contes

33. "Daar was eens een Houthakker met zyne vrouw, die zeven kinderen hadden, altemaal jongetjes. De oudste was maar tien jaar oud, en de jongste reeds zeven jaar. Men zal zich verbazen dat de houthakker zo veel kinderen gekreegen had in zo kort een tyd, maar de vrouw voer alle jaar naar de volewijk, en haalde d'er doorgaans twee te gelyk." (Ibid., p. 82)

34. Id.

35. L. M. De Meyer, De volks - en kinderprent in de Nederlanden van de $15^{\mathrm{C}}$ tot de $20^{\mathrm{e}}$ eeuw, Antwerpen, Standaardboekhandel, 1962, p. 495-499.

36. Voir Contes de ma Mère l'Oye. Vertellingen van Moeder de Gans, p. 53-54 et p. 83.

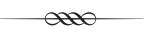


de la petite chaussette rouge, étant des contes de ma Mère l'Oye ${ }^{37}$, mince édition de format in-I2. La référence au conte Rood Kousje, déjà évoqué par J. Van Heemskerk, permet d'associer les contes de Perrault au folklore néerlandais. Outre les huit contes en prose de Perrault, l'édition comporte la traduction du conte L'Île de la Félicité de Madame d'Aulnoy et celle de L'Adroite Princesse de Mademoiselle Lhéritier. Cette traduction est peu soignée et quelquefois erronée. La Belle au bois dormant se perce la main avec des ciseaux (schaar) et non pas avec un fuseau. La citrouille de Cendrillon devient un citron (citroen) dans la traduction néerlandaise, les lézards deviennent des sauterelles (sprinkhanen) et les parents du Petit Poucet des bouchers (slagers), mais l'auteur adapte les contes au public enfantin. Il élimine des passages considérés moralement équivoques (la référence à la nuit de noces de la Belle au bois dormant disparaît complètement) et il remplace les moralités complexes de Perrault, qui permettent souvent un clin d'œil amusé au lecteur adulte ${ }^{38}$, par de véritables leçons morales pour enfants.

Une autre traduction néerlandaise paraît en Belgique sous le titre Contes amusants pour enfants, du Poucet, de La Belle au bois dormant et de nombreux autres contes pleins d'esprit. Servant de passe-temps amusant pour les jeunes enfants et autres lecteurs. Avec une moralité ajoutée à la fin de chaque conte pour la jeunesse. Augmentés du conte de Jantjen avec son cerf-volant. Ornés d'images ${ }^{39}$. Le texte dans ce recueil in-I2 est adapté ici ou là afin d'être relié aux illustrations, des gravures sur bois naïves provenant du fonds populaire. En plus des huit contes en prose de Perrault, le recueil comporte trois autres contes, à savoir Persinette de Charlotte-Rose Caumont de La Force, le conte moral Jantjen met zyn vliegerken et le fabliau Van het Smedjen. Les deux traductions sont rééditées plusieurs fois. Les contes de Perrault deviennent des classiques de la littérature enfantine, tout comme ceux, plus tardifs, des frères Grimm et d'Andersen, qui sont intégrés au répertoire enfantin au cours du XIX ${ }^{\mathrm{e}}$ siècle.

37. Vermaaklyle vertellingen van den ouden tyd, of zoogenaamde sprookjes van het Rood Kousje; zynde vertellingen van Moeder de Gans. Uit het Fransch vertaald. Met zede-lessen en aanmerkingen verrykt, $2^{\mathrm{e}}$ éd., Amsterdam, Erven de Weduwe van Jacobus van Egmont, ca. I780. Je n'ai pas pu trouver d'exemplaire de la première édition.

38. J.-P. Collinet, «Perrault et La Fontaine», The Romanic Review, 99, 3-4 (2008), p. 197.

39. Amusante kinder-vertellingskens, als van Duymken, de slaepende in den Bosch en veel andere geestige vertellingen. Dienende tot een vermaekelyk tyd-verdryf der jonge kinderen en andere personen. Benevens eene zedeleeringe gevoegt agter elk vertellingsken tot nut en voordeel der jonge jeugd. Vermeerdert met de vertellinge van Jantjen met zyn vliegerken, enz. Verciert met prenten, Gend, L. van Paemel, ca. I790.

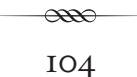


Or, à partir de la seconde moitié du XviII ${ }^{\mathrm{e}}$ siècle, le succès des autres contes français diminue. Inspiré par le succès du recueil Vermaaklyle vertellingen van den ouden tyd, of zoogenaamde sprookjes van het Rood Kousje, l'éditeur ajoute, vers 1790 , un second tome. Il annonce dans la préface de ce second tome qu'il compte y ajouter un troisième au cas où celui-ci s'avérerait être au goût du public. Contenant une traduction de plusieurs contes provenant des Illustres Fées, ce volume n'est pas réédité, contrairement au premier tome contenant les contes de Perrault, dont des rééditions paraissent en I805, I824, I826 et I827. Le troisième tome ne semble pas avoir été publié. Les éditions des contes de Madame d'Aulnoy se font également rares. Une seule autre traduction très littérale de deux contes semble, elle, avoir paru. Cependant, cette édition de I84I était de toute évidence adressée à un public adulte ${ }^{40}$. Il faut attendre la fin du XIX ${ }^{e}$ siècle pour que paraisse une nouvelle traduction des contes de Madame d'Aulnoy pour enfants. L'auteur néerlandais Reinoudina de Goeje, écrivant sous le pseudonyme Agatha, retient seulement sept contes qui formeront le recueil Sprookjes uit de oude doos (Contes d'hier). La traduction d'Agatha est la troisième à être publiée aux Pays-Bas, mais la première à être clairement adaptée à un nouveau public : les enfants néerlandais. La sélection qu'elle opère et la façon dont elle adapte les contes individuels laissent entrevoir quelques-unes des idées personnelles de la traductrice, notamment en matière de littérature enfantine et d'éducation, mais l'édition donne également quelques indices sur la réception des contes de Madame d'Aulnoy aux Pays-Bas.

\section{Reinoudina de Goeje devient Agatha ${ }^{4 \mathrm{I}}$}

Reinoudina de Goeje est la fille d'un pasteur protestant; cependant, dès le $\mathrm{XIX}^{\mathrm{e}}$ siècle, la critique remarque le manque d'intérêt porté à la religion dans ses livres. Ainsi l'auteur Johannes Hoek (I8I3-1893) se demande, dans le journal littéraire néerlandais De Tijdspiegel (Le Miroir du temps), si Agatha a délibérément omis les éléments chrétiens dans son roman pour

40. [M.-C. d'Aulnoy], Vertellingen der toovergodinnen, 2 vol., 's Gravenhage, Nederlandse Maatschappij der Schoone Kunsten, I84I. L'édition comporte des traductions de Gracieuse et Percinet et de La Grenouille bienfaisante.

4I. Une partie de l'analyse qui suit a été publiée sous une autre forme dans D. M. Hoogenboezem, «Marvel, Feminism and Reason: Rewriting Marie-Catherine d'Aulnoy's Fairy Tales for Dutch Children", H. Brown et G. Dow (eds), Readers, Writers, Salonnières: Female Networks in Europe, European Connections series, Oxford, Peter Lang, 20II, p. 259-275.

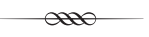


jeunes filles Eva Strooveld (I865) ${ }^{42}$. En effet, les phénomènes surnaturels ont moins d'attrait pour Agatha que l'éducation et la raison. Ces idées sont également reflétées dans ses contes. R. de Goeje elle-même reçoit une éducation usuelle. On lui donne des cours de catéchisme et de musique, et elle réalise des travaux d'aiguille. Cependant, sa correspondance montre qu'en outre Agatha et ses sœurs apprennent le français, l'anglais et l'allemand de leur père et de leurs frères. Dans une lettre non datée à son frère Bernard Elias (1834-1856), Agatha écrit, dans un français très approximatif :

Je me réjouis beaucoup que tu viendras encore un an ici avant d'aller à l'académie. Je pense que nous nous amuserons beaucoup. J'ai l'espérance d'être instruit de toi dans l'anglais. J'ai dû cesser d'apprendre parce que Ma [Maman] ne voulut pas que j'employerais mon temps pour cela ${ }^{43}$.

Plusieurs lettres de la mère de R. de Goeje, Wilhelmina Bernardina von Schilling (?-I872), montrent qu'elle est ravie lorsque sa fille l'aide dans les tâches ménagères ou s'applique aux travaux manuels. Ces lettres suggèrent qu'elle souhaite restreindre les occupations de sa fille aux travaux féminins les plus traditionnels. Or R. de Goeje apprend trois langues étrangères et, à l'âge de 26 ans, elle commence à traduire des livres pour enfants. Sa première traduction, Ruth en hare vriendinnen (Ruth et ses amies), écrite en collaboration avec sa sœur cadette, Anna Catherina Elisabeth (I839I858), est publiée en I859 sous le nom de sa sœur ${ }^{44}$. R. de Goeje emploie son pseudonyme pour toutes ses publications ultérieures, ainsi que dans sa correspondance avec les éditeurs. Agatha utilise l'argent qu' elle gagne pour aider sa mère, qui est devenue veuve mais prend soin de six enfants habitant encore avec elle. Dans une lettre à l'éditeur Suringar, écrit en I86I, Agatha évoque cette situation difficile lorsqu'elle lui demande quelques ouvrages à traduire : "Très volontiers je me rendrais utile de cette façon puisqu'il n'est probablement pas nécessaire de vous expliquer les difficultés que ma mère rencontre souvent pour nous pourvoir tous des choses

42. "Ik heb het recht niet aan de Schrijfster te vragen of zij opzettelijk of onwillekeurig de godsdienst in haar boek buiten werking heeft gelaten en alleen ongodsdienstige, - beter gezegd misschien: godsdienstlooze personen geschilderd heeft.» [Je n'ai pas le droit de demander à l'Écrivaine si elle a omis délibérément ou involontairement la religion dans ses livres et a peint seulement des personnages irréligieux ou plutôt des personnages sans religion.] (J. Hoek, «Letterkunde: Eene poging om meisjes te leeren dat Deugd Geluk is», De Tijdspiegel, I866, p. 74)

43. Lettre non datée à B. Elias, archives familiales.

44. A. C. E. de Goeje, Ruth en hare vriendinnen, een verhaal voor jongen meisjes, Haarlem, Zwaardenmaker, I859.

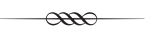


nécessaires ${ }^{45}$.» Dans la même lettre Agatha essaye de convaincre l'éditeur en mentionnant non seulement les ouvrages qu'elle a déjà traduits, mais également le fait que son frère Michaël Jan, qui avait obtenu en I858 son doctorat en lettres sémitiques, était prêt à l'aider : "Mon frère, qui a un doctorat en lettres, voudrait entreprendre la correction et donc je peux vous assurer que vous recevrez mes traductions toujours promptement et contrôlées avec précision ${ }^{46}$.»

En outre sa correspondance révèle que, malgré le fait qu'elle ait besoin d'argent, Agatha met en question la qualité de certains ouvrages qu'elle reçoit à traduire et qu'elle refuse quelquefois. Outre de nombreuses traductions, Agatha publie également un nombre important d'ouvrages de sa plume. Une bibliographie complète n'a pas encore été établie, mais elle comporterait de nombreux livres de jeunesse, des traductions et des adaptations des contes de Perrault, des frères Grimm et de Jeanne-Marie Leprince de Beaumont ${ }^{47}$, ainsi que des romans historiques, dont des romans sur la révolte des Pays-Bas (I568-I648) et la bataille de Waterloo, et des biographies de femmes célèbres des Pays-Bas ${ }^{48}$.

Agatha s'intéresse vivement à la position de la femme dans la société, et participe au débat féministe naissant à travers des écrits comme Onze werkkring (Notre cercle de travail, i868) et Jongejuffrouw Onnut (Mademoiselle Inutile, I876), dans lesquels elle décrit plusieurs opinions sur la question de l'émancipation et encourage les femmes à chercher un emploi approprié. Agatha est également la rédactrice en chef de l'un des

45. "Zoo heel gaarne maakte ik mij nuttig op deze wijze daar ik u wel niet zal behoeven te zeggen hoe moeilijk het mijne moeder dikwijls valt om ons allen van het nodige te voorzien." (Lettre à G. T. N. Suringar, le I3 août I86I, Bibliothèque universitaire d'Amsterdam)

46. "Mijn broeder die dr. In de Letteren is, belast zich voor mij gaarne met de correctie en zoo durf $i k$ u verzekeren dat u mijn werk steeds binnen de kortst mogelijke tijd en zeer nauwkeurig nagezien zult terug ontvangen." (Id.)

47. Agatha publie plusieurs adaptations des contes de Perrault, dont Sprookjes van Moeder de Gans (Les contes de ma Mère l'Oie), Leiden, Van den Heuvell en Van Santen, I866 et Alkmaar, Kluitman, I887; Het huis van Roodkapje’s grootmoeder [1893] (La maison de la grand-mère du Petit Chaperon rouge), Amsterdam, Jacs. G. Robbers; Asschepoetster [1893] (Cendrillon), Amsterdam, Jacs. G. Robbers. Elle traduit plusieurs contes des frères Grimm : Sprookjes voor de huiskamer en het huisgezin (Contes pour le séjour et la famille), 6 t., Schiedam, Roelants, I89o et elle écrit plusieurs versions du conte La Belle et la Bête de Leprince de Beaumont : Mooi Elsje [1874] (Belle petite Élise), Amsterdam, J. Vlieger, Een oud sprookje [1878] (Un vieux conte), Leiden, W. A. Sijthoff, et Belle en de beer [1893] (Belle et l'ours), Amsterdam, Jacs. G. Robbers .

48. De Pages van de Baron de Montigny: een verhaal uit de tachtig jarigen oorlog, Leiden, Van den Heuvell en Van Santen, I862; De Vrijwilliger: een verhaal van den slag bij Waterloo voor jongelieden, Leiden, D. Noothoven van Goor, 1865; Beroemde Nederlandsche Vrouwen, Leiden, Van den Heuvell en Van Santen, I869. 
premiers journaux féministes des Pays-Bas, Ons Streven (Notre aspiration, I870-1878). Outre des articles sur le débat féministe international, ce journal propose des résumés d'articles politiques publiés dans les journaux nationaux, et offre ainsi au public féminin des informations auparavant accessibles presque exclusivement aux lecteurs masculins ${ }^{49}$.

Agatha n'adapte pas uniquement les contes des auteurs mentionnés plus haut, elle traduit aussi les ouvrages de plusieurs autres, dont la romancière anglaise Charlotte-Mary Yonge (I823-I9OI), qui partage son intérêt pour l'éducation féminine et fonde une école pour femmes dont le nom, Mother Goose Society, rappelle le célèbre recueil de Perrault. De plus, il y a Joseph Wilfrid Ernest Gabriel Legouvé (I807-I903), défenseur de l'éducation pour femmes et enfants, et les auteurs allemands Emma Laddey (I84I-I892) et Eugénie John Marlitt (I825-I887) ${ }^{\text {so }}$. Cette esquisse nécessairement rapide de l'œuvre d'Agatha laisse entrevoir l'intérêt de l'auteur pour l'éducation et le débat féministe naissant.

Plusieurs comptes-rendus positifs et les nombreuses rééditions de ses livres attestent de leurs succès. Agatha est même invitée à écrire un roman pour la série intitulée Bibliotheek van Nederlandse schrijfsters ("Bibliothèque des auteurs féminins néerlandais", I878-I88I), tout comme des auteurs plus connus actuellement comme Elise van Calcar (I822-I904), (I83II9I5) et Virginie Loveling (I836-I923) ${ }^{\text {sI }}$. La collection s'avère un échec et quelques volumes seulement ont été effectivement publiés, mais le projet montre qu'Agatha est un auteur respecté à l'époque.

49. L. Jensen a analysé les premiers journaux féministes néerlandais dans son étude Bij uitsluiting geschikt voor de vrouwelijke sekse, Vrouwentijdschriften en journalistes in Nederland in de achttiende en negentiende eeuw, Hilversum, Verloren, 200I, p. I94.

50. Agatha a traduit les ouvrages de nombreux auteurs internationaux dont quelques-uns seulement peuvent être mentionnés ici. P's and Q's, or the Question of Putting upon (1872), de C. M. Yonge, est traduit par Agatha sous le titre de Pauline, een verhaal voor meisjes (Amsterdam, Jan Leendertz, 1873); Flitter und Gold (1873), de E. Laddey, est publié sous le titre Leonie en Elise, $2^{\mathrm{e}}$ éd. (Amsterdam, T. J. van Holkema, I887). E. Laddey est également mentionnée dans l'introduction du livre Op eigen wieken drijven: Novellen uit het Hoogduitsch (Schiedam, Van Dijk en Comp., I872). Nos filles et nos fils (I875), de J. W. E. G. Legouvé, parait sous le titre Tooneeltjes uit het familieleven (Tiel, H. C. A. Campagne, 1879). Thüringer Erzählungen (1869), de E. John (Marlitt), est traduit par Agatha et publié sour le titre Vertellingen naar het Hoogduitsch (Arnhem, Nijhoff, 1870).

5I. L. Kuitert, "Het debacle van een negentiende-eeuwse "vrouwenreeks". Bibliotheek van Nederlandse Schrijfsters", dans Digitale Bibliotheek voor de Nederlandse Letteren en ligne : http:// www.dbnl.org/tekst/kuitoozdebaoI_oI/kuitoo3debaoI_oI_ooor.php (2 avril 20II).

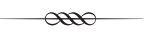




\section{Un conte pour enfants selon Agatha}

Des vingt-cinq contes de fées écrits par Madame d'Aulnoy, Agatha n'en sélectionne que sept pour son recueil Sprookjes uit de oude doos. Cette sélection laisse entrevoir déjà quelques-unes de ses préférences. Elle omet les contes plus ambigus contenant des scènes violentes ou présentant des relations amoureuses non conformistes. Le recueil d'Agatha ne comporte que deux contes précédemment publiés par J. D. Beman, à savoir De blauwe vogel (L'Oiseau bleu) et De oranjegeele dwerg (Le Nain jaune). Les autres contes qu'elle ajoute au recueil sont Gracieuse en Percinet (Gracieuse et Percinet), De prinses met de gouden lokken (La Belle aux cheveux d'or), Prinses Finette (Finette Cendron), De hinde in het bosch (La Biche au bois) et Het witte poesje ( $L a$ Chatte blanche). Cependant ces contes ne correspondent pas entièrement à ce que Agatha considère comme des histoires propres à être lues par des enfants.

Afin de rendre les contes plus facilement compréhensibles par son jeune public, Agatha omet la plupart des références à la France du XVII ${ }^{\mathrm{e}}$ siècle. La version de Madame d'Aulnoy contient de nombreuses allusions au champ sociopolitique, telles que des références à des événements historiques et à des personnalités comme Louis XIV, ainsi que du vocabulaire propre au XVII ${ }^{\mathrm{e}}$ siècle. Dans son conte La Biche au bois par exemple, Madame d'Aulnoy renvoie au palais du Roi Soleil :

Après cela elles prièrent la reine d'entrer dans leur palais, dont on ne peut faire une assez belle description : elles avaient pris pour le bâtir l'architecte du Soleil. Il avait fait en petit ce que celui du Soleil est en grand ${ }^{52}$.

Dans la version d'Agatha, cette référence explicite au château de Versailles disparaît tout comme la plupart des autres références à la société française.

Une autre caractéristique des contes de Madame d'Aulnoy est les références intertextuelles. Elle ajoute de nombreuses allusions à la mythologie, dont l'histoire de Psyché et Cupidon, qui avait été popularisée par Jean de La Fontaine, ainsi que des références intertextuelles aux romans baroques de Madeleine de Scudéry, aux Fables de La Fontaine et aux contes de fées de Perrault. Dans La Belle aux cheveux d'or par exemple, Madame d'Aulnoy renvoie aux fables lorsqu'elle décrit la rencontre du héros Avenant avec la carpe, nommée "Ma commère la carpe». La Fontaine utilise le mot "commère" pour évoquer une carpe et plusieurs autres animaux dans ses fables.

52. Madame d'Aulnoy, Contes nouveaux ou les Fées à la mode, N. Jasmin (éd.), Paris, Champion, 2008 , p. II5. 
Avenant adresse également la parole au corbeau en disant "Monsieur du Corbeau ", rappelant ainsi la fable Le Corbeau et le Renard.

Dans la version d'Agatha, les noms des personnages mythologiques disparaissent tout comme la plupart des références intertextuelles. Agatha ne préserve que celles aux contes de Perrault, devenus des classiques de la littérature enfantine depuis la seconde moitié du XVIII ${ }^{\mathrm{e}}$ siècle et dont Agatha avait elle-même écrit plusieurs adaptations. Ainsi, dans La Chatte blanche, le palais de l'héroïne est si beau qu'il n'en existe que dans des contes de fées. "Aussi le prince pense-t-il immédiatement au conte de La Belle au bois dormant, ainsi qu'à d'autres histoires qu'on lui avait racontées pendant sa jeunesse ${ }^{53}$.» À l'intérieur du palais de la chatte blanche, il découvre des tableaux représentant "des scènes du conte du Chat botté, le portrait d'une chatte, des chats chassant des rats ou des souris et puis plusieurs autres études sur la vies des chats ${ }^{54}$ ». Dans le conte Prinses Finette, Agatha ajoute également de nouvelles références à des textes connus de son jeune public. Pour consoler ses sœurs, Finette raconte le conte du pêcheur et de sa femme provenant des Kinder- und Hausmärchen (I8I7I82I) des frères Grimm. Dans le même conte, l'ogresse a été remplacée par une méchante reine portant un manteau de glace assise au milieu d'une chambre de neige, et rappelant la reine des neiges de Hans Christian Andersen (I844) 55 . Finette sauve ses deux sœurs ingrates en les caressant, tout comme la petite Gerda arrive à faire dégeler le cœur de Kay. Agatha omet toutes les références aux salons parisiens à l'exception des allusions aux contes de Perrault, mais elle se montre sensible au jeu intertextuel typique des contes français et ajoute des références plus ou moins explicites à d'autres contes connus de son public.

Agatha simplifie également les formulations : elle remplace les descriptions abstraites par des exemples concrets et elle raccourcit certains contes. Une autre façon de simplifier l'expression est d'employer des dialogues au lieu du discours indirect. Agatha développe l'emploi du discours direct surtout des personnages féminins, ce qui peut être considéré comme un

53. "Hopende dat hij daar beschutting voor den regen en misschien een nachtverblijf zou kunnen vinden stapte hij in de richting van het licht voort en bevond zich spoedig voor de poort van een kasteel, zoo mooi als slechts in de tooversprookjes voorkomt. De prins dacht dan ook dadelijk aan "de schoone slaapster in het bosch" en andere vertellingen die min hem in zijn jeugd gedaan had." (Agatha, Sprookjes uit de oude doos, Tiel, Campagne, 1882, p. 156)

54. "Zonderling schenen hem de onderwerpen gekozen — hier waren verschillende tooneelen uit het sprookje van de gelaarsde kat, daar het portret van een poes, aan de overzijde katten op ratten - of muizenjacht en verder allerlei studies uit het poezenleven.» (Ibid., p. 159)

55. Agatha, Sprookjes uit de oude doos, ouvr. cité, p. 90-92.

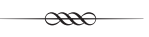


signe d'émancipation féminine, selon Ruth Bottigheimer ${ }^{56}$. Agatha ne remplace pas seulement la plupart des passages en discours indirect; elle ajoute également de nouvelles conversations. Ainsi, lorsque le premier ambassadeur arrive à la cour de la Belle aux cheveux d'or, cette princesse refuse d'épouser le roi. Madame d'Aulnoy rapporte ses propos en style indirect : «[...] elle répondit à l'ambassadeur qu' elle remerciait le roi, et qu'elle n'avait point envie de se marier ${ }^{57}$ ». Or, dans la version d'Agatha, la réponse est rapportée en style direct : «[...] elle s'écrie immédiatement: "Oh non merci! Je n'ai pas du tout envie de me marier" ${ }^{8}$ ". Agatha développe ainsi le caractère "féministe» des contes de Madame d'Aulnoy.

\section{Beauté naturelle, éducation et raison}

Agatha transforme les contes de Madame d'Aulnoy en contes pour enfants. Son jeune public paraît même à l'intérieur du recueil comme le public par excellence des contes. De nouveaux passages viennent complémenter la fin heureuse des contes L'Oiseau bleu, La Biche au bois et La Chatte blanche, dans lesquels, après le mariage des héros, ceux-ci racontent leurs propres aventures à leurs enfants. Les adaptations d'Agatha attestent d'une nouvelle vision de la famille comme refuge des enfants. Dans Le Nain jaune, la reine offre sa fille au nain en voyant approcher les lions, criant : "Monseigneur le Nain, Toute Belle est à vous [...] ne la refusez pas, c'est la plus charmante princesse de l'univers ${ }^{59}$." Dans la version d'Agatha, la reine ne peut se résoudre à abandonner sa fille, et elle dit seulement : "Sauvez-moi ${ }^{60}$.» De même, dans le conte Finette Cendron, la méchante reine, prête à abandonner ses trois filles sans le moindre remords, est métamorphosée en mère aimante qui est forcée à abandonner ses enfants.

Dans les contes de Madame d'Aulnoy, les descriptions de magie, de richesses inimaginables et les portraits de princesses d'une beauté irréelle abondent, mais Agatha préfere la beauté naturelle et elle souligne l'importance de l'éducation et de la raison. Dans sa version de La Belle aux cheveux d'or, la princesse ne porte plus de talons, et les diamants et les perles ornant sa robe disparaissent, tandis qu'Agatha préserve les fleurs

56. R. B. Bottigheimer, Grimm's Bad Girls and Bold Boys, New Haven et Londres, Yale University Press, 1987.

57. Madame d'Aulnoy, Contes des fées, ouvr. cité, p. I42.

58. Agatha, Sprookjes uit de oude doos, ouvr. cité, p. 29.

59. Madame d'Aulnoy, Contes des fées, ouvr. cité, p. 594.

6o. Agatha, Sprookjes uit de oude doos, ouvr. cité, p. I38.

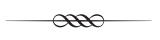


naturelles qu'elle porte dans les cheveux. Elle reproche à la mère de Toute Belle, héroïne du conte Le Nain jaune, de ne point corriger la vanité de sa fille. Aussi le nain dit-il à la reine : "Quoi de plus périssable que la beauté. Ma mère m'a dit que j'étais moi-même un bel enfant [...], ce qui importe c'est le cœur et non pas le visage ${ }^{6 \mathrm{r}}$." Les idées de la traductrice transparaissent aussi dans ses autres ouvrages. Ainsi Agatha avait utilisé son adaptation du conte Cendrillon pour plaider contre les corsets. Le motif du corset est présent dans la version originale de Perrault, mais Agatha insiste sur le thème. Elle ridiculise la vanité des deux sœurs méchantes, écrivant que la cadette ne pouvait guère rire puisque son corset était trop serré $^{62}$. Dans sa réécriture du conte La Belle et la Bête, l'héroïne s'achète des robes plus solides afin de bien pouvoir travailler ${ }^{63}$. Le dédain d'Agatha pour la vanité se montre également dans une de ses nombreuses lettres à son frère Michaël Jan, dans laquelle elle critique le caractère d'une jeune fille de leur connaissance avant d'enchaîner sur les qualités qu'une épouse devrait avoir :

Je conviens qu'elle est belle fille, qu'elle a la taille fine et mignonne mais en outre tu dois convenir qu'elle est très coquette quoiqu'encore enfant, très variable et très stupide. Elle est la voisine de Lise à l'école mais elle ne fait que copier l'ouvrage de notre petite Els. [...] Ce n'est pas que je pense que les savantes feront le bonheur de leurs époux mais pour une personne qui aime l'étude [...] comme toi il faut une épouse qui sache parler et juger de toutes sortes de choses ${ }^{64}$.

La préférence d'Agatha pour l'éducation et la raison est également reflétée dans ses contes. Dans sa version de La Biche au bois, le premier don des fées à la jeune princesse est l'intelligence, au lieu de la vertu chez Madame d'Aulnoy. Le malheur du vœu suivant lequel la princesse devra vivre, pendant quinze ans, sans voir la lumière du jour est atténué par le fait que son emprisonnement dans un château sans fenêtres lui laisse beaucoup de temps pour apprendre toutes sortes de choses. La princesse

6I. "Wat is vergankelijker dan schoonheid. Mijne moeder heeft mij verteld dat ik ook eens een mooi kind was, [...] en het komt meer op het hart aan dan op het gezicht." (Id.)

62. " "Ha, ha, een Asschepoester op het bal van den Prins!" riep de jongste, en zij trachtte te lachen, maar dat ging moeilijk omdat zij zo stijf ingeregen was. " ["Ha, ha, un Cendrillon au bal du prince", criait la cadette, et elle essayait de rire, mais cela était difficile parce que son corset était lacé si étroitement.] (Agatha, Sprookjes van Moeder de Gans, ouvr. cité, I866, p. I2)

63. "En ik", sprak Bella, "zal eten koken en al het huiswerk doen", en zij kocht zich sterke grove japons, die er beter tegen konden dan de dunne kleedjes, die zij gewoon was te dragen." ["Et moi", disait Bella, "je ferai la cuisine et tous les travaux ménagers", et elle s'achetait des robes plus résistantes que les robes délicates qu'elle portait d'habitude.] (Agatha, Een oud sprookje, ouvr. cité, page non numérotée.)

64. Lettre non datée à Michaël Jan, archives familiales.

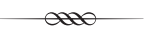


Finette et ses sœurs doivent étudier, elles aussi, alors que dans la version française il n'est question que de belles robes et de fêtes.

Dans ses contes, Agatha peint un monde plus rationaliste et quelquefois elle élimine des personnages magiques et des événements surnaturels. Dans La Belle aux cheveux d'or, l'un des personnages magiques est le chien parlant Cabriole. Il accompagne le héros Avenant lors de son voyage et l'aide à accomplir les tâches que la princesse lui impose. Ainsi il encourage le héros à faire une promenade en disant : "Mon cher maitre, je vous prie, ne désespérez point de votre bonne fortune; vous êtes trop aimable pour n'être pas heureux; allons dès qu'il fera jour au bord de la rivière ${ }^{65}$. Agatha réduit le rôle du chien dans sa version du conte. Elle remplace cette conversation par une description des pensées d'Avenant : "Après il se leva et sortit. La chambre lui devenait trop oppressante. Il lui semblait qu'il entendait déjà les courtiers se moquer de lui et qu'il voyait le regard déçu du roi ${ }^{66} . "$ Les conversations d'Avenant avec son chien sont remplacées par des monologues intérieurs du héros, offrant un portrait psychologique approfondi. Dans Finette Cendron, Agatha élimine la fée-marraine. C'est en réfléchissant que l'héroïne trouve des solutions aux problèmes qu'elle doit surmonter et, dans La Biche au bois, le portrait du prince ne parle pas par magie comme dans la version originale, mais grâce à une mécanique.

En outre Agatha supprime des détails violents. Dans la version française de La Belle aux cheveux d'or, Avenant tue un aigle pour sauver la vie d'un corbeau. Dans la version néerlandaise, le personnage tire avec des cartouches à blanc. Le corbeau s'échappe dans la fumée et l'aigle sort indemne. Agatha adapte également la punition d'Avenant, qui est condamné à mourir de faim dans la version française. Or, dans l'adaptation néerlandaise, le personnage est seulement mis au pain et à l'eau. Pour le conte La Chatte blanche, Agatha écrit une nouvelle fin, qui ressemble un peu au dénouement du conte La Belle et la Bête de Madame Leprince de Beaumont. Dans la version originale de La Chatte blanche, le prince tue l'animal, or le héros du conte d'Agatha refuse de tuer son amie la chatte et accepte même de l'épouser. C'est la fidélité du prince qui permet de mettre un terme à l'enchantement.

Contrairement à Madame d'Aulnoy, Agatha souligne les moralités dans sa version des contes, mais elle développe une thématique chère à

65. Madame d'Aulnoy, Contes des fées, ouvr. cité, p. I48.

66. "[...] na een paar uren gerust te hebben stond hij op en ging naar buiten. Het werd hem te eng in de slaapkamer, waar het hem gedurig was als hoorde hij hoe de hovelingen hem bespotten en hoe de koning hem teleurgesteld aankeek.» (Agatha, Sprookjes uit de oude doos, ouvr. cité, p. 35) 
la conteuse : le statut de la femme dans la société. Agatha met en scène des princesses intelligentes et actives tout comme Madame d'Aulnoy, et quelquefois elle développe le rôle des femmes au profit de celui des personnages masculins. Ainsi dans Le Nain jaune, la Fée du Désert, opposante du couple héroïque dans la version originale, devient l'héroïne de l'histoire au lieu du prince. Elle sauve les protagonistes et organise leur mariage, offrant une fin heureuse au conte.

\section{Conclusion}

La comparaison des premières traductions néerlandaises des contes de Madame d'Aulnoy et de Perrault permet de tracer l'évolution du livre de jeunesse aux Pays-Bas au cours des XviII ${ }^{\mathrm{e}}$ et $\mathrm{XIX}^{\mathrm{e}}$ siècles. Malgré le fait que la traduction de J. D. Beman soit adressée de façon explicite à un public jeune et que l'on y retrouve quelques reflets du débat sur l'éducation (valeur des contes et des récits historiques dans l'éducation des enfants), tout porte à croire que l'édition vise principalement un lectorat adulte. Les références à la jeunesse et aux leçons morales des contes font partie du jeu littéraire de l'auteur. La traduction néerlandaise atteste de la volonté de rendre les contes de fées français déjà très prisés plus accessibles à un vaste public.

Contrairement au recueil de J. D. Beman, la première traduction des contes de Perrault s'adresse véritablement à un public jeune et a une visée didactique : l'apprentissage du français et du néerlandais. Le succès de l'édition s'explique en partie par ce but très explicite, mais aussi par la qualité de la traduction. Les contes de Perrault deviennent aussitôt des classiques de la littérature enfantine, tout comme ceux des frères Grimm, d'Andersen et de Madame Leprince de Beaumont. Or la popularité des contes de Madame d'Aulnoy diminue fortement à partir de la seconde moitié du XVIII ${ }^{e}$ siècle. Les éditions pour adultes se font rares, et dans la littérature de jeunesse ses contes sont éclipsés par ceux de Perrault, des Grimm et d'Andersen.

Dans le recueil Sprookjes uit de oude doos, Agatha réécrit sept contes de Madame d'Aulnoy en s'adressant de façon exclusive aux enfants. La variété des sources d'Agatha atteste de son intérêt pour l'éducation et pour le débat sur le rôle de la femme dans la société, thématiques que l'on retrouve également dans les contes de Madame d'Aulnoy. Ce sont sans doute ces aspects des contes de fées français qui ont mené Agatha à les adapter pour la jeunesse néerlandaise. Or, si elle s'inspire souvent des 
ouvrages d'autres auteurs, ses choix impliquent rarement une adhésion complète. Elle adapte plus qu'elle ne traduit. Agatha simplifie l'expression, raccourcit les contes et omet les références à la France du XVII ${ }^{\mathrm{e}}$ siècle. Cependant elle se montre sensible au jeu intertextuel. Elle préserve les références aux contes de Perrault et ajoute même des allusions à d'autres textes connus de son jeune public, dont les contes des frères Grimm et d'Andersen. En outre, ses adaptations laissent entrevoir ses conceptions pédagogiques. Elle souligne l'importance de la famille et de l'éducation et atténue les scènes violentes. Le pays des fées de Madame d'Aulnoy, avec ses palais impossibles et ses richesses inimaginables, est remplacé par un monde moins exotique et plus rationaliste. Or si Agatha en souligne les moralités, elle ne réduit pas les contes pétillants de la conteuse à de simples contes moraux ennuyeux. Elle préserve et développe ce qui concerne le rôle de la femme. La plupart des héroïnes actives et intelligentes de Madame d'Aulnoy ont des qualités identiques dans la version néerlandaise, et souvent elles expriment leur point de vue de manière plus convaincante que dans l'original. L'adaptation d'Agatha témoigne d'une vision pédagogique assez progressiste, et permet donc de montrer l'évolution de la littérature de jeunesse, mais elle annonce également les lectures féministes des contes de Madame d'Aulnoy par la critique moderne. 